
\title{
25 Research Soure \\ ERIL: An Algorithm for Emotion Recognition From Indian Languages Using Machine Learning
}

PRAMOD MEHRA ( $\sim$ pramodmehra11@gmail.com )

CIPET - Lucknow: Central Institute of Petrochemicals Engineering \&amp; Technology - Lucknow

\section{Parag Jain}

Roorkee Institute of Technology, Roorkee, Uttarakhand, India

\section{Research Article}

Keywords: MFCC, LPC, Pitch, Indian Speech, Emotion recognition, emotion classification, Catboost

Posted Date: April 23rd, 2021

DOl: https://doi.org/10.21203/rs.3.rs-449758/v1

License: (9) This work is licensed under a Creative Commons Attribution 4.0 International License. Read Full License 


\section{Abstract}

For a human interaction with machine, it is important that it understand the mood of the speaker. Until now we train machines on neutral speeches or utterances. The mood of a person would affect their performances. Deciphering human mood is challenging for the machines, as human can create fourteen distinct sound in a second. For a machine to understand the human behaviour, it should understand the acoustic abilities of the human ear. Mel Frequency Cepstral Coefficients (MFCC) and Linear Prediction coefficients (LPC) can replicate human auditory system. The proposed model Emotion Recognition from Indian Languages (ERIL) extracts emotions like fear, anger, surprise, sadness, happiness, and neutral. ERIL first pre-processes the voice signal, extracts selective MFCC, LPC, pitch, and voice quality features, then classifies the speech using Catboost. ERIL is a multilingual emotion classifier, it is independent of any language. We checked it on Hindi, Gujarati, Marathi, Punjabi, Bangla, Tamil, Oriya, and Telugu. We recorded a speech dataset of various emotions in these languages. ERIL is compared to other benchmark classifiers.

\section{Full-text}

Due to technical limitations, full-text HTML conversion of this manuscript could not be completed. However, the manuscript can be downloaded and accessed as a PDF.

\section{Figures}
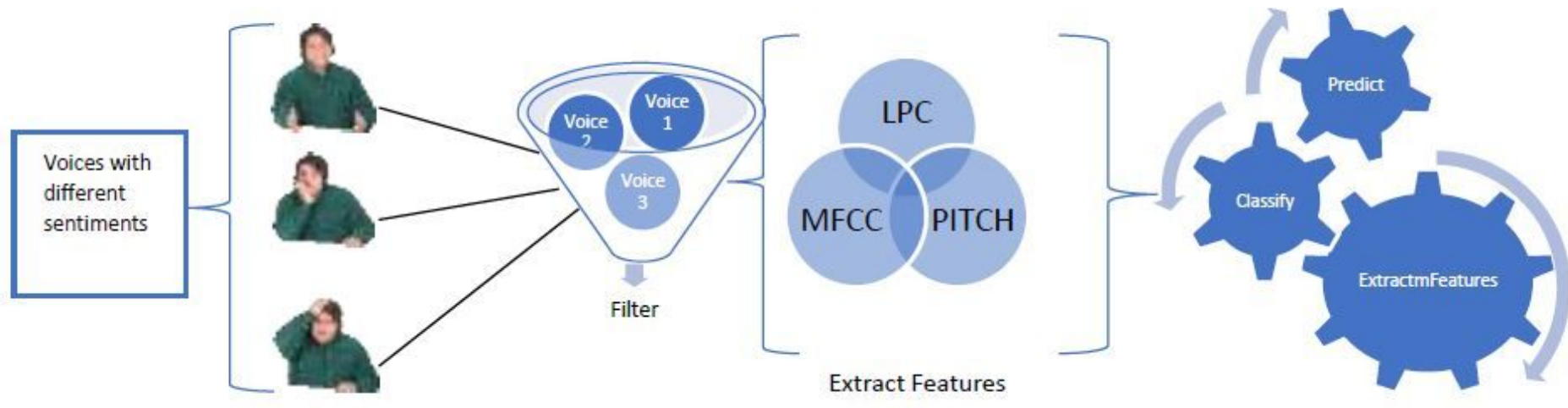

Catboost

\section{Figure 1}

Proposed Model 


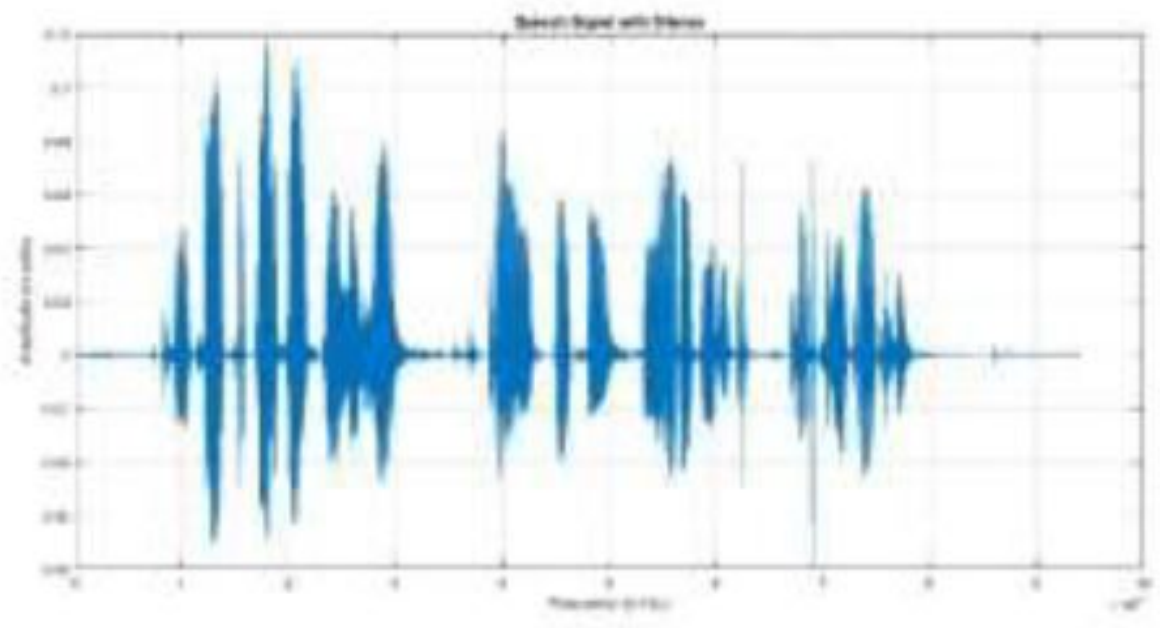

Figure 2

Original voice

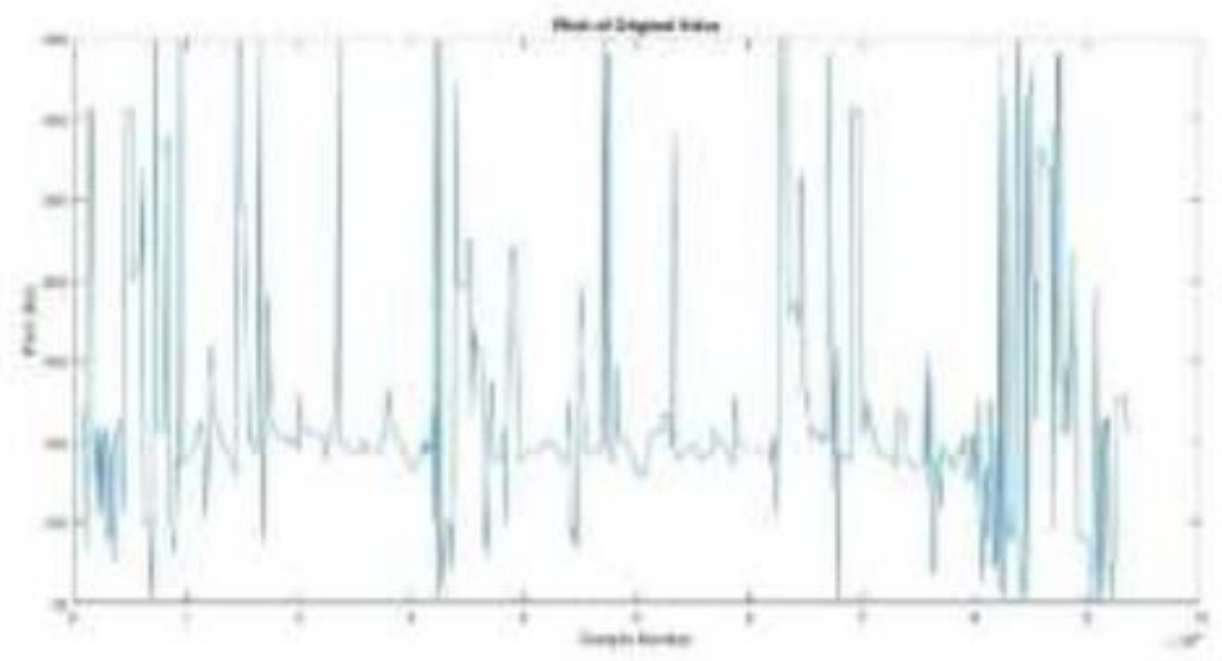

Figure 3

Pitch estimation plot

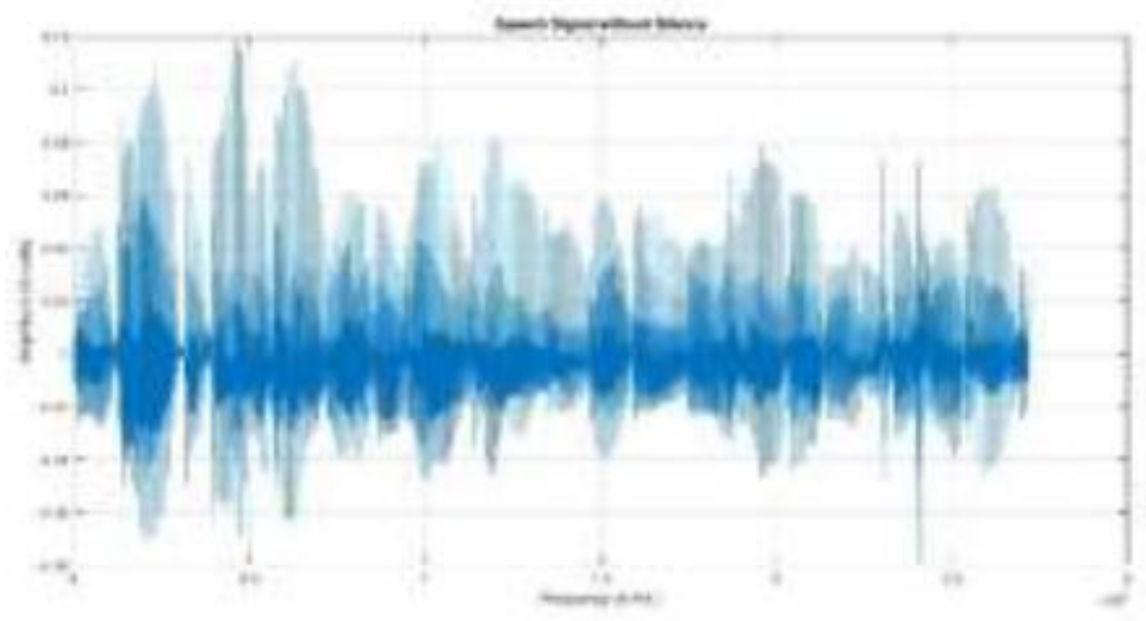

Page 3/6 
Figure 4

\section{Filtered voice}

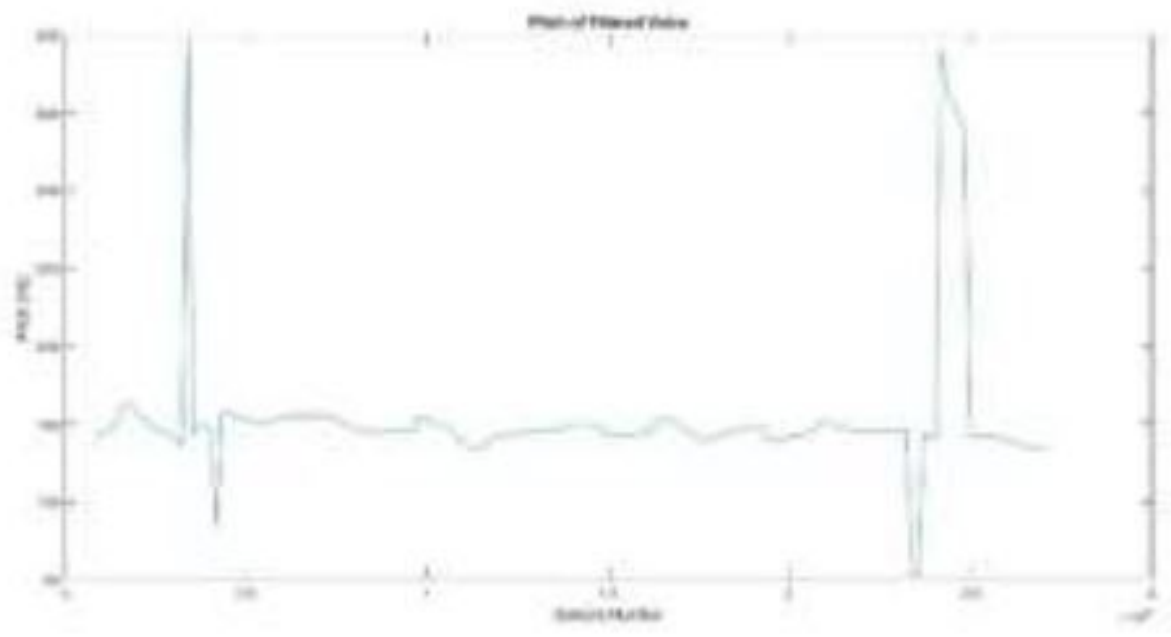

Figure 5

Effect of Windowing

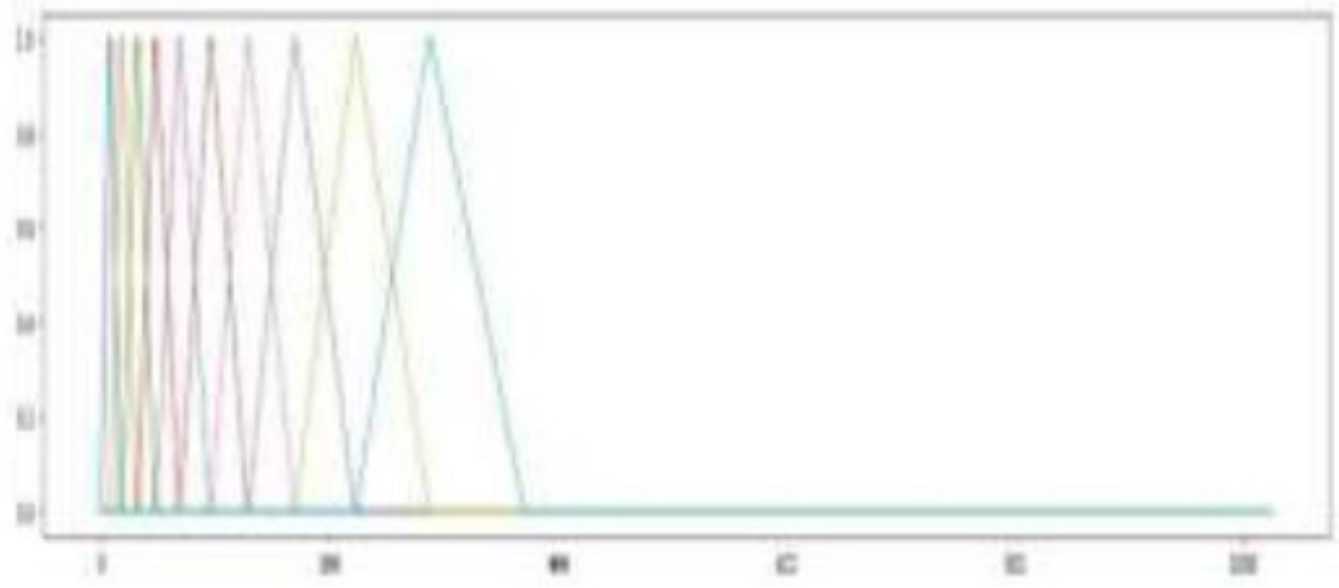

Figure 6

Plot of the filter points 


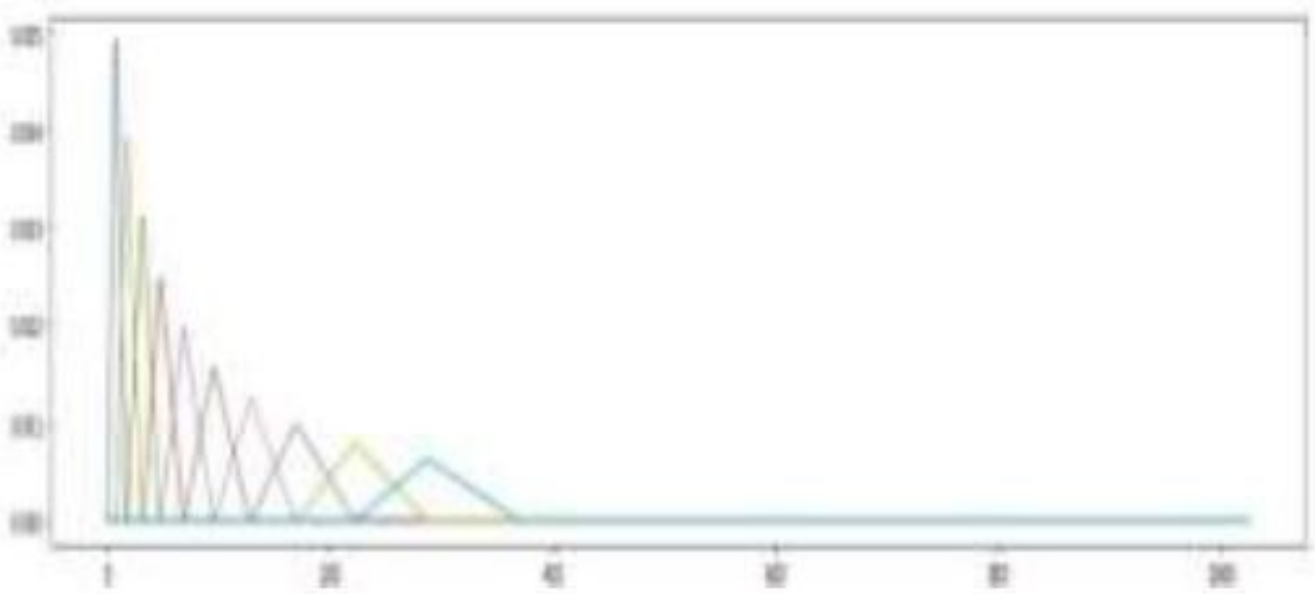

Figure 7

Plot of Mel bands calculated

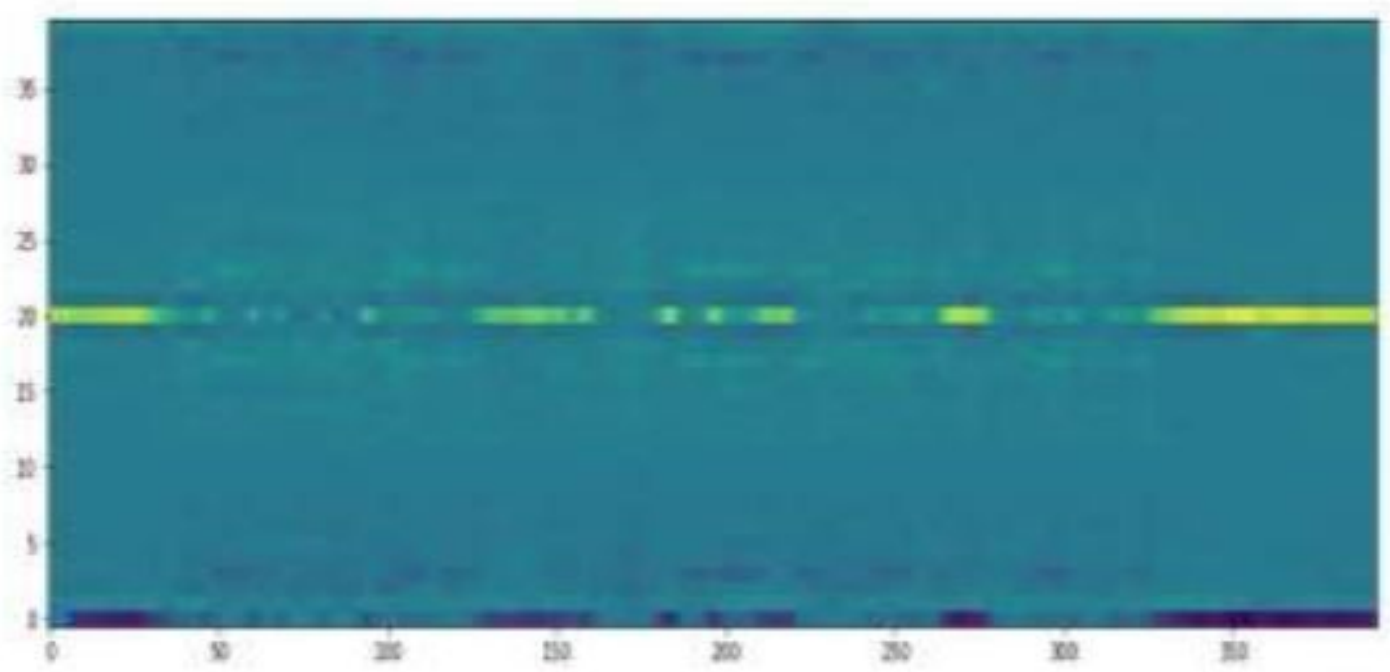

Figure 8

Spectrogram plot of the second derivative of MFCC 


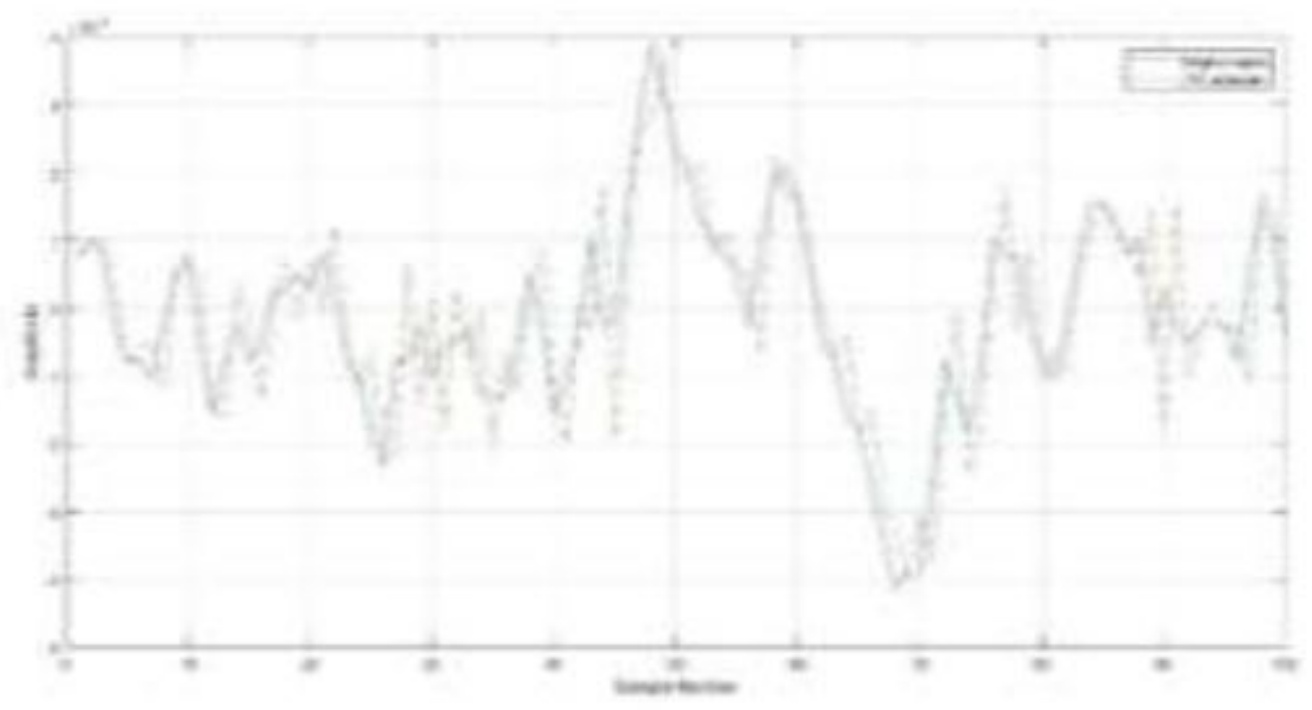

Figure 9

LPC plot with filtered voice

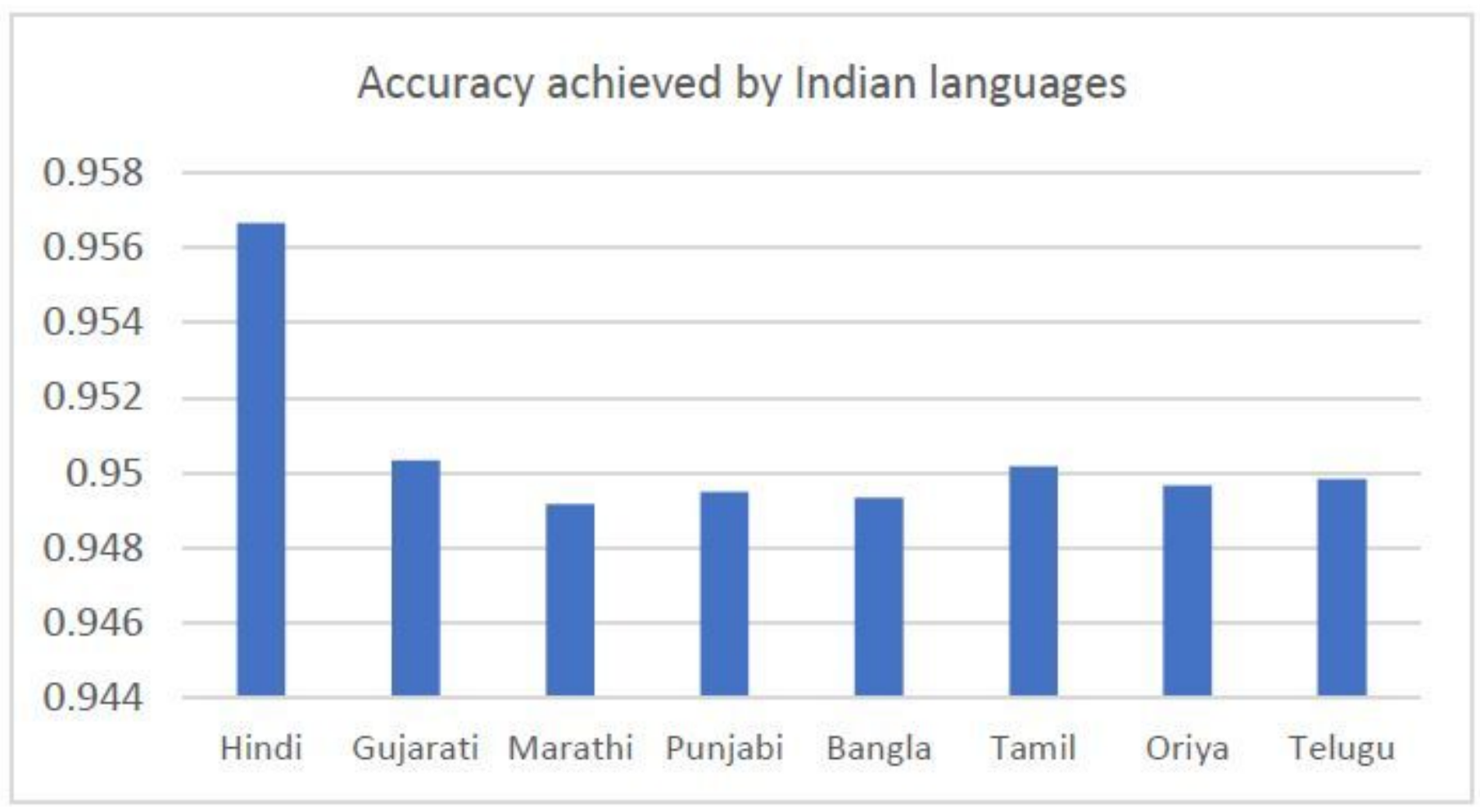

Figure 10

Based on table 6 a plot is drawn of contribution of each language in the accuracy. 